\title{
The self-consistent determination of HF electroconductivity of strongly coupled plasmas
}

\author{
V. A. Srećkovića , V. M. Adamyan ${ }^{\mathrm{b}}$, Lj. M. Ignjatovića ${ }^{\mathrm{a}}$ A. A. Mihajlov ${ }^{\mathrm{a}}$ \\ ${ }^{a}$ Institute of Physics, Pregrevica 118, Zemun, Belgrade, Serbia \\ ${ }^{b}$ Department of Theoretical Physics, Odessa National University, 65026 Odessa, Ukraine
}

\begin{abstract}
Here is presented the calculation of the dynamic electrical conductivity of fully ionized, strongly coupled plasmas as a function of the external electric field frequency $\omega$. The calculations are based on the the formula for the energy-dependent collision frequency which is determined by means of the Green function theory methods, as a sum over the Matsubara frequencies. The domain of extremely high electron density: $10^{21} \leq n_{e} \leq 10^{24} \mathrm{~cm}^{-3}$, and for the temperature varying from $10 \mathrm{kK}$ to $1.000 \mathrm{kK}$ was examined. The real and imaginary parts of the conductivity for every electron density are presented in the generalized Drude-like form as a two-parameter function of the frequency $\omega$ in the region $0<\omega<0.5 \omega_{p}$, where $\omega_{p}$ is the plasma frequency. A good agreement between the obtained results and the existing theoretical and computing simulation data is shown.
\end{abstract}

Key words: dynamic electrical conductivity, fully ionized, strongly non-ideal

\section{Introduction}

The defining of the dynamic high frequency (HF) electrical conductivity of the strongly coupled plasma is an actual and very important problem because such characteristics of plasma as the HF dielectric function, the coefficients of refractivity and reflectivity, and skin depth are expressed just in terms of the dynamic electrical conductivity. However, until recently the determination of the dynamic plasma conductivity in the wide ranges of the electron densities $\left(n_{e}\right)$ and the temperatures $(T)$ was not taken as the priority task. Namely, in the most cases the mentioned problem was not investigated as the main one [1-5], and only few authors presented the results of the direct definition of HF electrical conductivity [6-10] related to some selected values of $n_{e}$ and $T$.

Because of that, a few years ago a certain research of the HF conductivity of the plasmas was started. It was thought that the previous researches of the transport properties of the dense strongly coupled plasmas free of any external fields [11, 12], and the plasmas in the presence of the constant magnetic field [13], gave some results which were useful in the case of the plasma in the external time depending fields. From this reason the research of the HF plasma conductivity was also devoted to the dense strongly coupled plasmas. The main aim was to examine the HF conductivity in the whole area of such plasmas, including here the region of the extremely large degrees of its non-ideality. This was especially important, since the existing literature data about the HF plasma conductivity [6-10] are just related to the extremely strongly coupled plasmas $\left(10^{21} \mathrm{~cm}^{-3}<n_{e} \lesssim 10^{24} \mathrm{~cm}^{-3}\right)$. 
The first results of that research were presented in [14], where the new method of the calculations of the strongly coupled plasma HF conductivity is described. This method was tested on the plasmas with $10^{17} \mathrm{~cm}^{-3} \leq n_{e} \leq 10^{19} \mathrm{~cm}^{-3}$ and $10^{4} \mathrm{~K} \leq T \leq 5 \cdot 10^{4} \mathrm{~K}$, in the microwave and far-infrared regions of $\omega$, were $\omega$ is the frequency of the external electric field. The developed method uses the energy-dependent electron collision frequency which is determined as a sum over the Matsubara frequencies, using the Green function theory methods and the corresponding self-consistent calculations procedure, which is described in details in [11, 13]. The final results, i.e. the HF conductivity $\sigma(\omega) \equiv \sigma\left(\omega ; n_{e}, T\right)$, as well as its real and imaginary parts $\sigma_{R e, I m}(\omega) \equiv \sigma_{R e, I m}\left(\omega ; n_{e}, T\right)$, were presented in the adequate, three-parameter Drude-like form. As the second step, by [15] the same method with its improved numerical procedure it was applied to the denser plasmas, with $10^{19} \mathrm{~cm}^{-3} \leq n_{e} \leq 10^{21} \mathrm{~cm}^{-3}$ and $2 \cdot 10^{4} \mathrm{~K} \leq T \leq 10^{6} \mathrm{~K}$, in infrared, visible and near UV regions of $\omega$.

Since, in [14, 15] the domain of low and moderately non-ideal plasmas was covered, the first aim of this Letter was to apply the developed method by the adequate numerical procedure, in order to determine of the HF conductivities of the strongly and extremely coupled plasmas, i.e. for $10^{21} \mathrm{~cm}^{-3} \leq n_{e} \lesssim 10^{24} \mathrm{~cm}^{-3}$. This way would not only laboratory strongly coupled plasmas but also some astrophysical plasmas (for examples, the photospheres of some white dwarfs [16]) be considered.

Another aim is to compare the obtained results with the existing theoretical results and computer simulation data [6-10], which refer to $n_{e}=3.8 \cdot 10^{21} \mathrm{~cm}^{-3}, 2.52 \cdot 10^{22} \mathrm{~cm}^{-3}$ and $1.61 \cdot 10^{24} \mathrm{~cm}^{-3}$. Such a comparison is very important because there is no data concerning the direct experimental measurements of HF conductivity of the dense strongly coupled plasmas [4], [17].

Similarly to our previous papers [14, 15], the HF conductivity $\sigma(\omega)$, as well as $\sigma_{R e, I m}(\omega)$, are presented here in the three-parameters Drude-like form, where one of these parameters is the static conductivity $\sigma_{0}$ of the considered plasma, which is suitable for further consideration. However, while in [14, 15] the values of $\sigma_{0}$ could be taken from [13], the corresponding values for the region $10^{21} \mathrm{~cm}^{-3} \leq n_{e} \lesssim 10^{24} \mathrm{~cm}^{-3}$ were especially calculated here.

The calculations of $\sigma_{R e}(\omega)$ and $\sigma_{I m}(\omega)$ in all considered cases, for all taken values of $n_{e}$ and $T$, are performed here in the frequency range $0<\omega<0.5 \cdot \omega_{p}$, where $\omega_{p}$ is the plasma electron frequency. The results are compared with the data from [6-10].

\section{Model}

\subsection{Basic equations}

In this Letter it is considered a completely ionized plasma in a homogenous and monochromatic external electric field $\vec{E}=\overrightarrow{E_{0}} \cdot \exp \{-i \omega t\}$. According to [14], the dynamic electric conductivity of a strongly coupled plasma $\sigma(\omega)=\sigma_{R e}(\omega)+i \sigma_{I m}(\omega)$ is presented by the expressions

$$
\begin{gathered}
\sigma(\omega)=\frac{4 e^{2}}{3 m} \int_{0}^{\infty} \frac{\tau(E)}{1-i \omega \tau(E)}\left[-\frac{d w(E)}{d E}\right] \rho(E) E d E \\
\sigma_{R e}(\omega)=\frac{4 e^{2}}{3 m} \int_{0}^{\infty} \frac{\tau(E)}{1+(\omega \tau(E))^{2}}\left[-\frac{d w(E)}{d E}\right] \rho(E) E d E \\
\sigma_{I m}(\omega)=\frac{4 e^{2}}{3 m} \int_{0}^{\infty} \frac{\omega \tau^{2}(E)}{1+(\omega \tau(E))^{2}}\left[-\frac{d w(E)}{d E}\right] \rho(E) E d E
\end{gathered}
$$


where $e, m$ and $E$ are the charge, mass and energy of the free electron, $\tau(E)$ - the relaxation time presented later in the text, $\rho(E)$ - one-electron states density in the energy space, $w(E)=$ $[\exp (\beta E-\beta \mu)+1]^{-1}$ - the Fermi-Dirac distribution function, $\mu$ - the chemical potential of the ideal gas of the free electrons with the density $n_{e}$ and the temperature $T$, and $\beta=\left(k_{B} T\right)^{-1}$.

Comparing Eq. (1) by $\omega=0$ with the expression for the static conductivity $\sigma_{0}$ of the fully ionized strongly coupled plasma from [11, 13], one can see that

$$
\sigma(\omega=0)=\sigma_{0}=\frac{4 e^{2}}{3 m} \int_{0}^{\infty} \tau(E)\left[-\frac{d w(E)}{d E}\right] \rho(E) E d E .
$$

Consequently, from Eqs.(2) and (3) it follows the validity of the relations $\sigma_{R e}(\omega=0)=\sigma_{0}$, $\sigma_{I m}(\omega=0)=0$, which have the sense of the conditions in the point $\omega=0$ during the numerical calculations of $\sigma_{R e}(\omega)$ and $\sigma_{I m}(\omega)$. The right side of Eq.(4) has the Lorentz-like form. Because of that the quantity $\tau(E)$ was interpreted in $[11,13]$ as the corresponding electron relaxation time (total electron-ion plus electron-electron), characterizing the considered plasma in the absence of the external fields. Since the same quantity $\tau(E)$ is used in the expressions (1-3) for $\sigma(\omega)$ just the good understanding of the static conductivity $\sigma_{0}$ as a function of $n_{e}$ and $T$ has a very important role in defining the strongly coupled plasma dynamic conductivity. Because of this fact our research was started in (see [14]) in the region of the plasma electron density $n_{e} \leq 10^{19} \mathrm{~cm}^{-3}$, where the basic method of the determination of the $\sigma_{0}$ from [11, 13] was experimentally verified [18].

Accordingly to $[11,13]$, the relaxation time $\tau(E)$ is defined in the self-consistent approximation by the following relations:

$$
\begin{aligned}
\tau^{-1} & =\frac{4 \pi m n_{e} e^{4}}{\beta(2 m E)^{3 / 2}} \int_{0}^{\sqrt{8 m E} / \hbar} \frac{d q}{q} \sum_{a, v} \frac{Z_{a}^{2} \Pi_{a v}(q)}{n_{a} \varepsilon_{v}^{3}(q)}, \\
\varepsilon_{v} & =1+\frac{4 \pi e^{2}}{q^{2}} \sum_{a, v} Z_{a}^{2} \Pi_{a v},
\end{aligned}
$$

where $a$ is the label for the plasma species, $Z_{a}$ - the species charge number, $\Pi_{a v}(q)$ - corresponding polarization operator in the random-phase approximation (a simple loop), $\varepsilon_{v}$ - the dielectric function expressed in terms of these quantities, and the $v$ summation is extended over the Matsubara even frequencies [11]. Here, the polarization operator $\Pi_{i(j) v}(q)$ of non-degenerate $j$-th ion component is equal to $\beta n_{i(j)} \delta_{v 0}$, and the RPA polarization operator $\Pi_{e v}(q)$ of partially degenerate free electron component, namely

$$
\Pi_{e v}^{R P A}(q)=\frac{m}{q}\left(\frac{k_{F}}{\pi \hbar}\right)^{2} \int_{0}^{\infty} \frac{u d u}{\exp \left(u^{2} / \theta-\beta \mu\right)+1} \ln \left|\frac{2 k_{F} u+q+i Q_{v}}{2 k_{F} u-q+i Q_{v}}\right|
$$

where $k_{F}=\left(3 \pi^{2} n_{e}\right)^{1 / 3}, Q_{v}=4 \pi v m / \hbar^{2} \beta q, v=0, \pm 1, \ldots$, and $\theta=(2 m / \beta) \cdot\left(\hbar k_{F}\right)^{-2}$.

The main numerical problems, which appear during the calculation of the static conductivity $\sigma_{0}$, are connected to the defining of the relaxation time $\tau$. Namely, the number of the members in the $v$ sum in Eq. (5), which has to be calculated individually, rapidly increases with the increase of the electron density $n_{e}$. For example, at $n_{e} \sim 10^{24} \mathrm{~cm}^{-3}$ this number is order of magnitude $\sim 10^{5}$, while each member itself has to be calculated by a special procedure. It is clear that the determination of the HF conductivity $\sigma(\omega)$, requires the volume of the calculations several times larger than in the case of the determination of $\sigma_{0}$. 


\subsection{Parameterized form}

According to [14] and [15], the quantities $\sigma_{R e}$ and $\sigma_{I m}$ can be presented in the parameterized form, suitable for the comparison with the corresponding Drude-like formulas, namely

$$
\sigma_{R e}(\omega)=\sigma_{0} \frac{1}{1+\left(\omega \tau_{0}^{*}\right)^{2} k_{1}^{2}}, \quad \sigma_{I m}(\omega)=\sigma_{0} \frac{\left(\omega \tau_{0}^{*}\right) k_{2}}{1+\left(\omega \tau_{0}^{*}\right)^{2} k_{1}^{2}},
$$

where $\sigma_{0}$ is given by Eq. (4), $\tau_{0}^{*}$ is the effective relaxation time defined by $\sigma_{0}=\frac{n_{e} e^{2}}{m} \tau_{0}^{*}$ and $k_{1} \equiv k_{1}(\omega)$ and $k_{2} \equiv k_{2}(\omega)$ are parameters, which describe the influence of the deviation of the $d w / d E$ from the $\delta$-function [13]. When the electron component of the considered plasma can be treated as a highly degenerate gas of the free electrons, both coefficients $k_{1}$ and $k_{2}$ tend to 1 , and equation (7) reduce to the Drude-Lorentz model [19]. Using the plasma frequency $\omega_{p}$ and the dimensionless parameter $f_{0 p}$, defined by relations $\omega_{p}=\left(4 \pi n_{e} e^{2} / m\right)^{1 / 2}, f_{0 p}=\omega_{p} \tau_{0}^{*}=4 \pi \sigma_{0} / \omega_{p}$, the parameterized expressions for $\sigma_{R e}(\omega)$ and $\sigma_{I m}(\omega)$ convert into

$$
\sigma_{R e}(\omega)=\sigma_{0} \cdot \frac{1}{1+\left(\omega / \omega_{p}\right)^{2} f_{0 p}^{2} k_{1}^{2}}, \quad \sigma_{I m}(\omega)=\sigma_{0} \cdot \frac{\left(\omega / \omega_{p}\right) f_{0 p} k_{2}}{1+\left(\omega / \omega_{p}\right)^{2} f_{0 p}^{2} k_{1}^{2}},
$$

Our previous numerical analysis have shown that $k_{1}$ and $k_{2}$ can be cast in the following approximate fitting form

$$
k_{j}=k_{j 0}-a_{j} \cdot \frac{a_{j} b_{j}\left(\omega / \omega_{p}\right)}{1+a_{j} b_{j}\left(\omega / \omega_{p}\right)}, \quad j=1,2,
$$

where the adjustment parameters $k_{10}, a_{1}, b_{1}$ and $k_{20}, a_{2}, b_{2}$ are determined numerically from the condition of the best relation between the 'exact' and fitted values of the factors $k_{1}$ and $k_{2}$. Let us notify that by just using the expressions (7)-(8) for $\sigma_{R e}(\omega)$ and $\sigma_{I m}(\omega)$ we get the possibility to confirm whether Drude-like model (when $k_{1,2} \approx 1$ ) has the physical sense for the considered $n_{e}, T$ and $\omega$.

\section{Results and discussion}

In this paper it was defined the dynamic conductivity $\sigma(\omega)$ of completely ionized hydrogenlike plasmas, when in expression (5) only two species (electron and ion with $Z=1$ ) are taken into account. Here we had on mind that the using of the expressions (7) and (8) for the determination of $\sigma_{R e}(\omega)$ and $\sigma_{I m}(\omega)$ require the knowledge of the static conductivity $\sigma_{0}$. From this reason the values of $\sigma_{0}$ are especially determined here in the considered domain $10^{21} \leq n_{e} \leq 10^{24} \mathrm{~cm}^{-3}$ and $10 \mathrm{kK} \leq T \leq 1000 \mathrm{kK}$. These values are presented in Tables 1

Then, according to [14] and [15], the coefficients $k_{1}$ and $k_{2}$ in Eqs. (7) and (8) are taken in the form (9), where they are expressed in terms of the parameters $k_{j 0}, a_{j}$ and $b_{j}, j=1,2$. The values of these parameters were also computed in the same domain $\left(10^{21} \leq n_{e} \leq 10^{24} \mathrm{~cm}^{-3}\right.$ and $10000 \mathrm{~K} \leq T \leq 1000000 \mathrm{~K}$ ) and presented in Table 2 and Table 3 .

The conduct of $\sigma_{R e}(\omega)$ and $\sigma_{I m}(\omega)$, calculated directly by Eqs. (2) and (3) for $10^{21} \leq n_{e} \leq$ $10^{24} \mathrm{~cm}^{-3}$ and $10 \mathrm{kK} \leq T \leq 1000 \mathrm{kK}$, is displayed in tables and figures in the ranges $0<\omega \leq 0.5$. $\omega_{p}$. The figures 1, 2, 4and 6, demonstrate the regular behavior of $\sigma_{R e}(\omega)$, i.e. the convergence to the corresponding values of $\sigma_{0}\left(n_{e}, T\right)$ when $\omega \rightarrow 0$, and the existence of the interval of variation of $\omega$ where $\sigma_{R e}(\omega)$ is practically constant. We observe the tendency of this interval to decrease when temperature $T$ increases. Similarly, the figures 3,5 and 6 , demonstrate a regular behavior of 
$\sigma_{\text {Im }}(\omega)$, i.e. the convergence to zero when $\omega \rightarrow 0$, and the presence of a maximum in the interval $0<\omega<0.5 \omega_{p}$. The obtained results, together with the data from [6], [9], [7] and [10], show that the position of this maximum comes to $0.5 \omega_{p}$ when $n_{e}$ grows in the region $n_{e} \leq 5 \cdot 10^{22} \mathrm{~cm}^{-3}$, and decrease from $0.5 \omega_{p}$ when $n_{e}$ increases in the region $n_{e}>5 \cdot 10^{22} \mathrm{~cm}^{-3}$.

We compare the behavior of $\sigma_{R e}(\omega)$ and $\sigma_{I m}(\omega)$, determined in this Letter, with the behavior of the corresponding quantities determined in: [6] and [7], for $\Gamma=0.5$ and $r_{s}=1$; [6], [7] and [8], for $\Gamma=0.5$ and $r_{s}=4$; [9] for $\Gamma=10$ and $r_{s}=1$; [10], for $n_{e}=3.8 \cdot 10^{21} \mathrm{~cm}^{-3}$ and $T=3.3 \cdot 10^{4} \mathrm{~K}$. Here $\Gamma=e^{2} /(a k T)$ and $r_{s}=a / a_{0}$ are the well-known non-ideality and Brueckner parameters, where $a_{0}$ and $a=\left[3 /\left(4 \pi n_{e}\right)\right]^{1 / 3}$ are the Bohr and the Wigner-Seitz radii. The results of [7], [8] and [10] were obtained by using the molecular dynamics (MD) simulation method, while the results of [6] and [9] are theoretical. The data from [6] are obtained by two analytical expressions for the collision frequency (the main one, obtained within the memory function formalism, and the approximate one found by the solution of the high-frequency hydrodynamic equation derived from the macroscopic equations of electron motion). The corresponding curves in the example $\Gamma=0.5$ and $r_{s}=1$ are shown in Figs. 1 and 3, and in the case $\Gamma=0.5$ and $r_{s}=4$ - in Figs. 4 and 5

The figures 1 and 3 show that in the example $\Gamma=0.5$ and $r_{s}=1$ our values of $\sigma_{R e}(\omega)$ and $\sigma_{\text {Im }}(\omega)$ are mostly in good relation with the results obtained in [7] by the MD simulation method, except the region $\omega \lesssim 0.1 \cdot \omega_{p}$. The same conclusion is valid for the theoretical results obtained in [6] for $\Gamma=0.5$ and $r_{s}=1$ by means of the mentioned approximate expression. Connected to the theoretical results from [6] and [9], obtained by means of the main analytical expression in the cases $\Gamma=0.5$ and $\Gamma=10$, for the same $r_{s}=1$, a good agreement with our results exists in the whole region of $\omega$. Therefore it cold be expected since that main analytical expression has significant similarity with our expressions.

From figure 3] concerning $\sigma_{I m}(\omega)$, one can see that not only our results, but both results of [6] significantly disagree with the results of the MD simulation from [7] in the region $\omega \lesssim 0.1 \cdot \omega_{p}$. We believe that final conclusions, respecting this fact, can be reached only after some additional MD simulations, since in this region the behavior of of the static conductivity $\sigma_{0}$ as a function of $n_{e}$ and $T$ has the dominant role, and till now we have been considering our values of $\sigma_{0}$ as fairly reliable.

In the example when $\Gamma=0.5$ and $r_{s}=4$ the figure 4 shows a good relation of our values of $\sigma_{R e}(\omega)$ with the values obtained in [6] and [8], since only in the region $\omega<0.1 \cdot \omega_{p}$ there exists some appreciable difference (the largest differences are less that 10 percents) between our results and those of [6]. The figure [5] concerning $\sigma_{I m}(\omega)$, shows a qualitative agreement (the differences are less or close to 20 percents) of our results with the results of [6] and [8].

\section{Conclusions}

The results obtained in this Letter, together with the ones presented in [14] and [15], make possible easy determination of $\sigma_{R e}(\omega)$ and $\sigma_{I m}(\omega)$ in very wide ranges of the electron density and temperature, namely $10^{17} \leq n_{e} \leq 10^{24} \mathrm{~cm}^{-3}$ and $10^{3} \mathrm{~K} \leq T \leq 10^{6} \mathrm{~K}$. Consequently, they can be used for the theoretical research, as well as for the interpretation of experimental data in the cases of high pressure discharge, capillary discharge, shock waves, etc., where the strongly coupled plasmas, including extremely dense plasmas, are created. Apart of that, $\sigma_{R e}(\omega)$ and $\sigma_{I m}(\omega)$ can be useful in some cases of stellar plasmas (e.g., white dwarfs). It is important that the presented results allow the determination of other optical characteristics of strongly coupled plasmas: the permeability, the refractivity, etc. The advantage of the method, which was used in 
our research until now, is that it does not contain any empirical parameters, and have got internal possibilities for its further improvement.

Let us emphasize that the used procedure is similar to the procedure for the determination of the conductivity tensor in the case of a strongly coupled plasma in an external static magnetic field [13]. This fact opens the possibility for the development of the method of determination of the conductivity of strongly coupled plasmas in an external high frequency electromagnetic field in the presence of the static (or quasi-static) external magnetic field.

Finally, the method used in this Letter does not assume in advance the quasi-Drude shape for $\sigma(\omega)$, but the way of presentation of the final expressions for $\sigma_{R e}$ and $\sigma_{I m}$ automatically demonstrates the character of the deviations from Drude's expression, at least in the case we consider.

Certainly, since the numerical procedure for the determination of $\sigma_{R e}(\omega)$ and $\sigma_{I m}(\omega)$ which is used here is quite complicated, it is desirable to develop some other methods which would allow an easier calculation of these quantities. Apart of that, in the lower part of the considered domain of the electron density $\left(n_{e} \leq 10^{20} \mathrm{~cm}^{-3}\right)$ it is necessary to develop the method of determination of the dynamic conductivity which would take into account the presence of the plasma neutral component.

\section{Acknowledgments}

The presented work is performed within the Project 141033 "Non-ideal laboratory and ionosphere plasma : properties and applications" financed by the Ministry of Science of the Republic of Serbia, as well as the INTAS (GSI-INTAS Project 06-1000012-8707).

\section{References}

[1] G. A. Kobzev, I. T. Iakubov, M. M. Popović (Eds.), Transport and Optical Properties of Nonideal Plasma, Plenum Press, New York and London, 1995, Ch.6.2.

[2] M. A. Berkovsky, Y. K. Kurilenkov, H. M. Milchberg, Phys. Fluids. B4 (1992) 2423.

[3] I. Morozov, H. Reinholz, G. Ropke, A. Wierling, G. Zwicknagel, ,Phys. Rev.E 71 (2005) 066408.

[4] H. Reinholz,Ann:Phys.Fr 30 (2006) 1.

[5] I. Kwon, L. Collins, J. Kress, N. Troullier, Phys. Rev.E 54 (1996) 2844.

[6] M. A. Berkovsky, D. Djordjević, Y. K. Kurilenkov, H. M.Milchberg, M. M. Popović, J. Phys. B: Mol.Opt.Phys. 24 (1991) 5043.

[7] L. Sjogren, J. P. Hansen, E. L. Pollock,Phys Rev A 24 (1981) 1544.

[8] H. Furukawa, K. Nishihara, M. Kawaguchi, H. Sakagami, T. Hiramatsu, H. Yasui, Particle Simulations on Static and Dynamic Properties of Two Component Hot Dense Plasmas, Japan:Elsevier, 1990.

[9] M. A. Berkovsky,Physica A 214 (1995) 461.

[10] H. Reinholtz, I. Morozov, G. Ropke, T. Millat,Phys Rev E 69 (2004) 066412-1.

[11] Z. Djurić, A. A. Mihajlov, V. A. Nastasyuk, M. Popović, I. M. Tkachenko, Phys. Lett. A 155 (6,7) (1991) 415.

[12] V. M. Adamyan, Z. Djurić, A. M. Ermolaev, A. A. Mihajlov, I. M. Tkachenko, J. Phys.D. 27 (1994) 927.

[13] V. M. Adamyan, Z. Djurić, A. M. Ermolaev, A. A. Mihajlov, I. M. Tkachenko, J. Phys. D 27 (1994) 111.

[14] A. A. Mihajlov, Z. Djurić, V. M. Adamyan, N. M. Sakan, J. Phys. D 34 (2001) 3139.

[15] V. M. Adamyan, Z. Djurić, A. A. Mihajlov, N. M. Sakan, I. M. Tkachenko, J. Phys. D 37 (2004) 1896.

[16] D. Koester, A\&AS 39 (1980) 401.

[17] H. Reinholtz, R. Redmer, G. Ropke, A. Wierling, Phys Rev E 62 (2000) 5648.

[18] Y. Vitel, M. E. Bezzari, A. A. Mihajlov, Z. Djurić, Phys. Rev.E 63 (2001) 026408.

[19] I. P. Shkarofski, T. W. Johnston, M. P. Bachymski, Partial kinetics of plasmas, New York, Addison-Westley, 1966. 
Table 1: The computed static plasma conductivity $\sigma_{0}$ as a function of the electron density $n_{e}$ and temperature $T$, $\left[10^{3}(\Omega \mathrm{m})^{-1}\right]$.

\begin{tabular}{cccccccc}
\hline \hline$T[K]$ & \multicolumn{7}{c}{$n_{e}\left[\mathrm{~cm}^{-3}\right]$} \\
\cline { 2 - 7 } & $10^{21}$ & $5 \cdot 10^{21}$ & $10^{22}$ & $5 \cdot 10^{22}$ & $10^{23}$ & $5 \cdot 10^{23}$ & $10^{24}$ \\
\hline 10000 & 28.56 & 78.23 & 122.1 & 388.0 & 677.7 & 2579 & 4016 \\
15000 & 33.90 & 86.68 & 135.7 & 410.4 & 701.0 & 2698 & 5175 \\
20000 & 40.17 & 93.34 & 145.2 & 430.8 & 723.2 & 2721 & 4886 \\
30000 & 54.19 & 108.2 & 160.8 & 464.6 & 762.6 & 2767 & 5037 \\
50000 & 85.37 & 145.7 & 198.4 & 511.8 & 824.2 & 2816 & 5070 \\
100000 & 174.2 & 257.6 & 320.1 & 636.6 & 948.7 & 2920 & 5070 \\
200000 & 382.8 & 516.8 & 606.6 & 985.9 & 1305 & 3200 & 5228 \\
500000 & 1165 & 1461 & 1639 & 2278 & 2726 & 4779 & 6669 \\
1000000 & 2806 & 3393 & 3727 & 4829 & 5531 & 8282 & 10430 \\
\hline
\end{tabular}

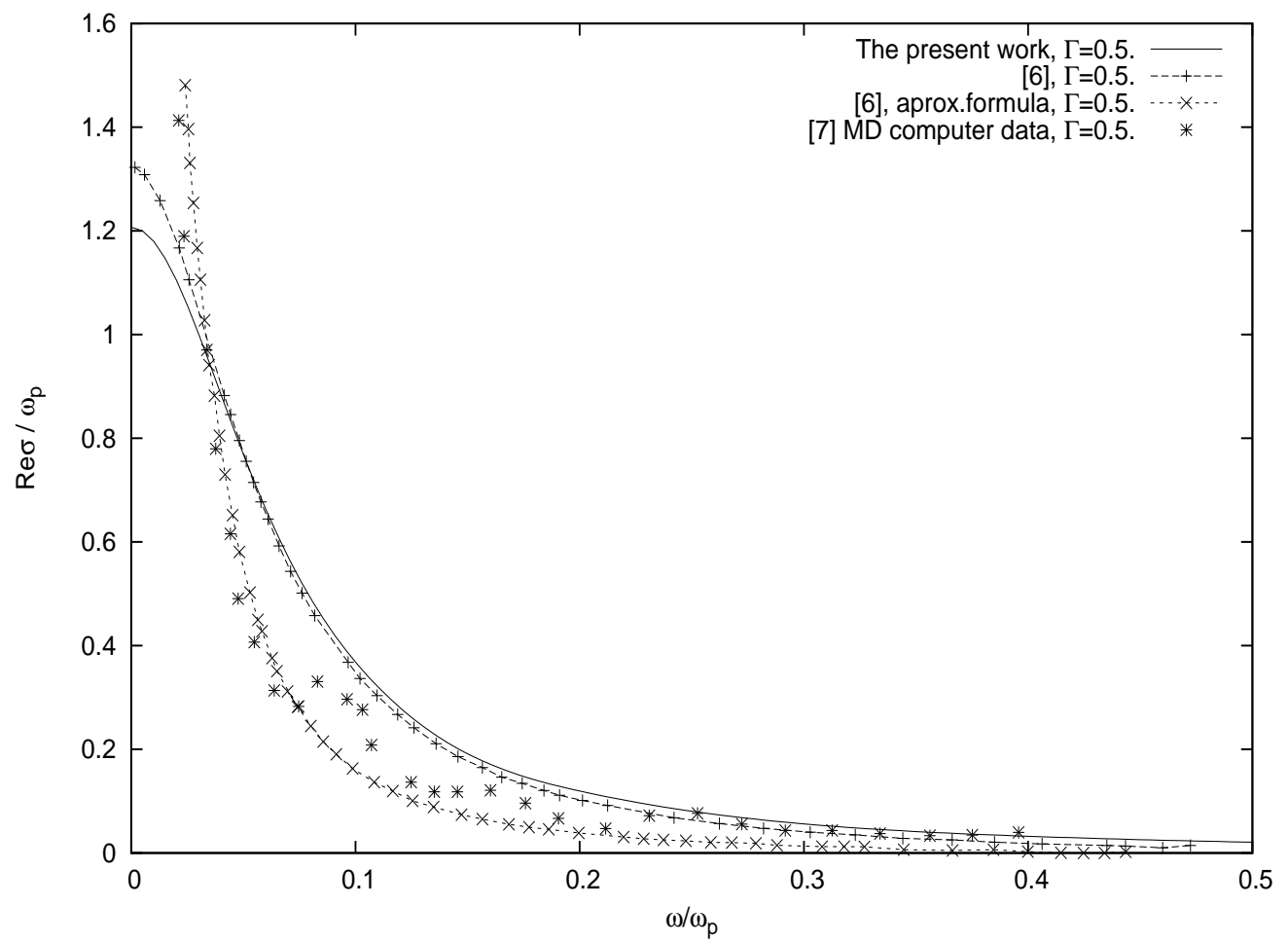

Figure 1: Plasma dynamic conductivity in [CGS] units, as a function of $\left(\omega / \omega_{p}\right)$ : for $\Gamma=0.5$ and $r_{s}=1$, with the results from [6] and [7], real part. 
Table 2: Parameters $k_{10}$ (first row for each $T$ ), $a_{1}$ and $b_{1}$ (second and third rows, respectively) as functions of $n_{e}$ and $T$.

\begin{tabular}{cccccccc}
\hline \hline$T[K]$ & \multicolumn{7}{c}{$n_{e}\left[\mathrm{~cm}^{-3}\right]$} \\
\cline { 2 - 8 } & $10^{21}$ & $5 \cdot 10^{21}$ & $10^{22}$ & $5 \cdot 10^{22}$ & $10^{23}$ & $5 \cdot 10^{23}$ & $10^{24}$ \\
\hline 10000 & 1.36558 & 1.14298 & 1.11647 & 1.04503 & 1.02252 & 1.03775 & 1.23393 \\
& 0.91630 & 0.42670 & 0.36160 & 0.11570 & 0.05374 & 0.00832 & 0.00572 \\
& 0.80560 & 1.46304 & 1.71715 & 9.66853 & 29.2500 & 447.500 & 975.700 \\
15000 & 1.56925 & 1.21020 & 1.14482 & 1.07335 & 1.04339 & 1.00815 & 0.96590 \\
& 1.12789 & 0.47940 & 0.35200 & 0.17550 & 0.09703 & 0.01560 & 0.00541 \\
& 1.06607 & 2.22226 & 2.84164 & 7.27586 & 17.5200 & 244.600 & 1032.00 \\
50000 & 1.70187 & 1.31099 & 1.19312 & 1.09268 & 1.06185 & 1.01567 & 1.02906 \\
& 1.22186 & 0.63100 & 0.40760 & 0.20970 & 0.13380 & 0.02620 & 0.01223 \\
& 1.40904 & 2.20497 & 3.36852 & 6.89132 & 13.5600 & 147.200 & 459.700 \\
50000 & 1.85027 & 1.50720 & 1.33432 & 1.12245 & 1.08882 & 1.02599 & 1.01296 \\
& 1.30102 & 0.90070 & 0.61770 & 0.24750 & 0.18120 & 0.04943 & 0.02285 \\
50000 & 2.22529 & 2.19804 & 3.11968 & 7.66151 & 11.4900 & 80.3700 & 252.400 \\
& 1.98074 & 1.74157 & 1.58508 & 1.21653 & 1.13706 & 1.05221 & 1.02846 \\
& 1.36853 & 1.13885 & 0.94640 & 0.37520 & 0.24640 & 0.09391 & 0.05013 \\
& 4.09404 & 3.00424 & 3.14523 & 7.67889 & 11.7600 & 44.5000 & 117.400 \\
50000 & 2.09206 & 1.95358 & 1.85948 & 1.51913 & 1.34490 & 1.10857 & 1.06780 \\
& 1.45707 & 1.31413 & 1.21608 & 0.79270 & 0.53610 & 0.17830 & 0.11230 \\
200000 & 9.00543 & 5.95428 & 5.25356 & 5.95785 & 8.79760 & 29.6900 & 56.2000 \\
& 2.16088 & 2.07644 & 2.02196 & 1.81532 & 1.67551 & 1.28276 & 1.16478 \\
& 1.53680 & 1.43764 & 1.37555 & 1.13930 & 0.96530 & 0.41640 & 0.24720 \\
500000 & 19.0300 & 12.0400 & 10.2000 & 7.99801 & 8.30448 & 19.0000 & 34.4800 \\
& 2.21152 & 2.16940 & 2.13967 & 2.03771 & 1.96910 & 1.70479 & 1.53717 \\
& 1.59191 & 1.54523 & 1.51208 & 1.39149 & 1.30924 & 0.97700 & 0.75090 \\
& 57.9900 & 32.3600 & 25.8200 & 16.9600 & 14.9600 & 14.5800 & 18.2200 \\
1000000 & 2.26912 & 2.20725 & 2.19266 & 2.12778 & 2.08724 & 1.93392 & 1.82624 \\
& 1.65679 & 1.58655 & 1.56883 & 1.49589 & 1.44904 & 1.26324 & 1.12668 \\
& 138.900 & 76.0200 & 59.2600 & 34.3800 & 28.2200 & 20.7800 & 20.2900 \\
\hline
\end{tabular}


Table 3: Parameters $k_{20}$ (first row for each $T$ ), $a_{2}$ and $b_{2}$ (second and third rows, respectively) as functions of $n_{e}$ and $T$.

\begin{tabular}{cccccccc}
\hline \hline$T[K]$ & \multicolumn{7}{c}{$n_{e}\left[\mathrm{~cm}^{-3}\right]$} \\
\cline { 2 - 8 } & $10^{21}$ & $5 \cdot 10^{21}$ & $10^{22}$ & $5 \cdot 10^{22}$ & $10^{23}$ & $5 \cdot 10^{23}$ & $10^{24}$ \\
\hline 10000 & 1.22578 & 1.09158 & 1.07647 & 1.02936 & 1.01445 & 1.03622 & 1.23283 \\
& 0.85360 & 0.43490 & 0.38030 & 0.11700 & 0.05381 & 0.00821 & 0.005572 \\
& 0.73820 & 1.23034 & 1.42388 & 9.08148 & 28.4700 & 451.400 & 1033 \\
15000 & 1.36175 & 1.12968 & 1.09216 & 1.04821 & 1.02833 & 1.00524 & 0.9649 \\
& 1.05294 & 0.44090 & 0.34460 & 0.17850 & 0.09769 & 0.01544 & 0.005332 \\
& 0.96230 & 2.16439 & 2.57804 & 6.67217 & 16.6700 & 246.000 & 1066 \\
5000 & 1.45309 & 1.19154 & 1.11970 & 1.06089 & 1.04061 & 1.01077 & 1.02666 \\
& 1.13563 & 0.56690 & 0.37520 & 0.21280 & 0.13520 & 0.02613 & 0.01221 \\
& 1.26874 & 2.20112 & 3.31066 & 6.26462 & 12.6900 & 145.600 & 457.6 \\
30000 & 1.55543 & 1.32016 & 1.20668 & 1.07872 & 1.05821 & 1.01668 & 1.0084 \\
& 1.19391 & 0.81940 & 0.55200 & 0.24180 & 0.18220 & 0.04959 & 0.02282 \\
50000 & 2.01639 & 2.10622 & 3.14718 & 7.16940 & 10.6200 & 77.8800 & 249.7 \\
& 1.64423 & 1.48078 & 1.37339 & 1.13410 & 1.08691 & 1.03422 & 1.01839 \\
& 1.23540 & 1.04367 & 0.86190 & 0.33860 & 0.23370 & 0.09454 & 0.05031 \\
& 3.76200 & 2.76140 & 2.98249 & 7.80417 & 11.4000 & 42.0900 & 113.6 \\
100000 & 1.71861 & 1.62588 & 1.56188 & 1.32945 & 1.21494 & 1.06960 & 1.04423 \\
& 1.29643 & 1.18484 & 1.10384 & 0.71430 & 0.47780 & 0.17160 & 0.1116 \\
& 8.46413 & 5.51045 & 4.84527 & 5.80599 & 8.93956 & 28.6500 & 53.33 \\
500000 & 1.76372 & 1.70824 & 1.67192 & 1.53184 & 1.43625 & 1.17662 & 1.10373 \\
& 1.36115 & 1.28090 & 1.23103 & 1.03249 & 0.87480 & 0.37330 & 0.2281 \\
500000 & 17.9300 & 11.3300 & 9.55919 & 7.45556 & 7.86760 & 19.4000 & 34.65 \\
& 1.79739 & 1.76921 & 1.74994 & 1.68242 & 1.63638 & 1.45691 & 1.34383 \\
& 1.40905 & 1.37086 & 1.34419 & 1.24460 & 1.17609 & 0.88420 & 0.677 \\
1000000 & 53.6500 & 30.0600 & 24.1100 & 15.9400 & 14.0400 & 13.9000 & 17.87 \\
& 1.82998 & 1.79479 & 1.78467 & 1.74212 & 1.71534 & 1.61265 & 1.53976 \\
& 1.45044 & 1.40480 & 1.39017 & 1.33254 & 1.29409 & 1.13771 & 1.01851 \\
& 130.200 & 70.5400 & 54.8300 & 31.8700 & 26.2700 & 19.5000 & 19.17 \\
\hline
\end{tabular}




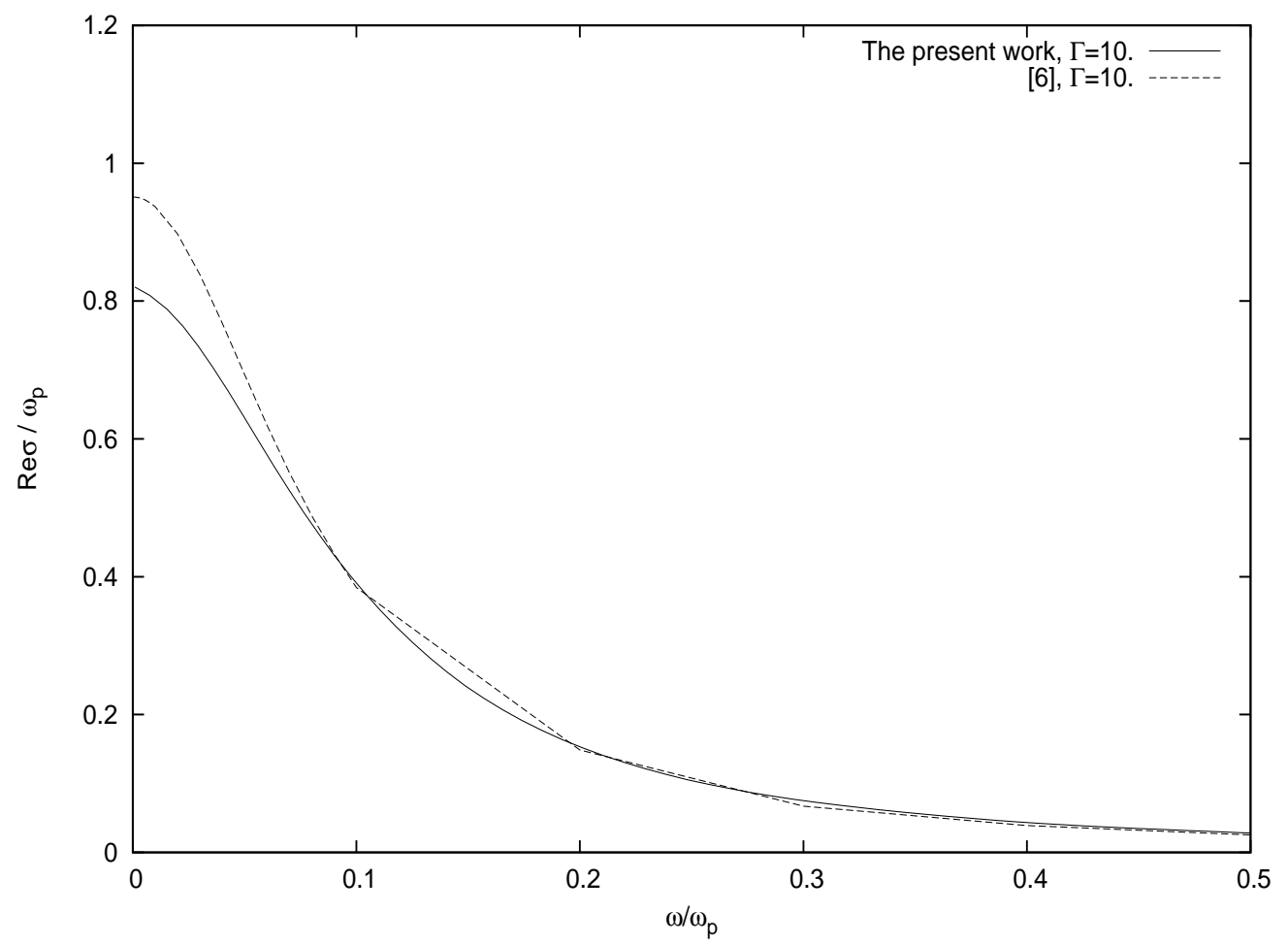

Figure 2: Plasma dynamic conductivity in [CGS] units, as a function of $\left(\omega / \omega_{p}\right)$ : for $\Gamma=10$ and $r_{s}=1$ with the results from [9], real part. 


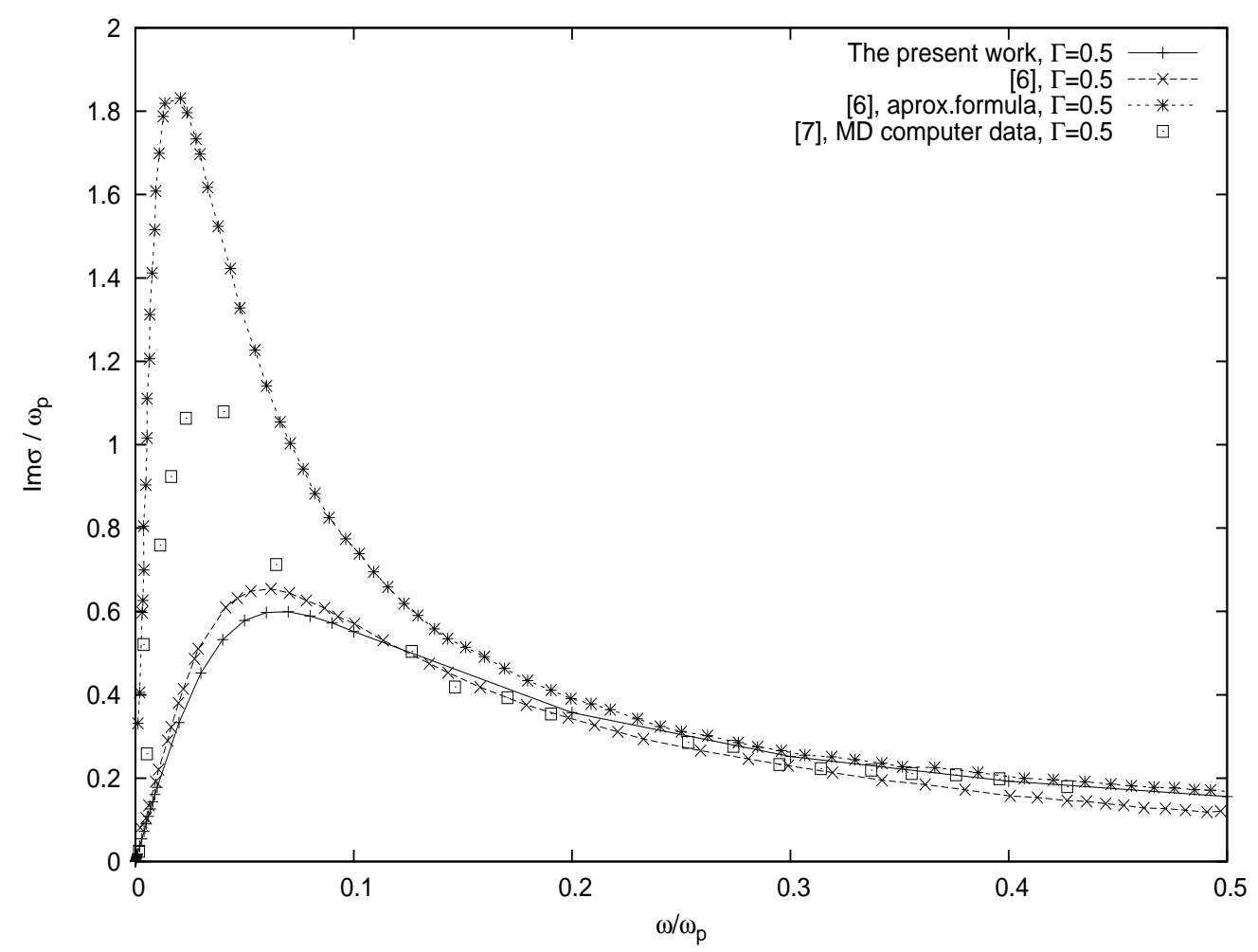

Figure 3: Same as in Fig. 1 but for imaginary part. 


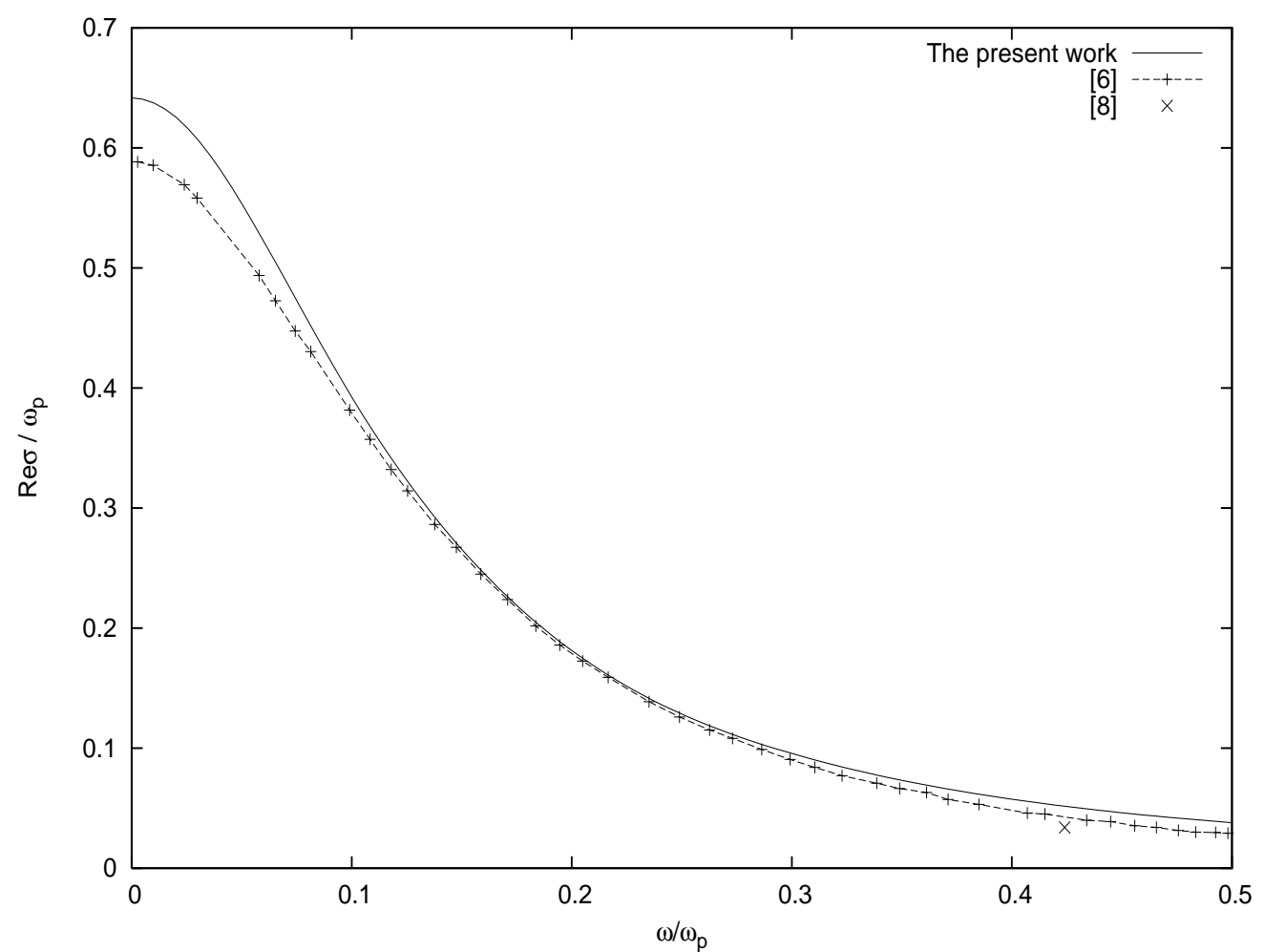

Figure 4: Plasma dynamic conductivity in [CGS] units for $\Gamma=0.5$ and $r_{s}=4$, as a function of $\left(\omega / \omega_{p}\right)$ frequency ratio, together with results of other authors [6] and [8], real part. 


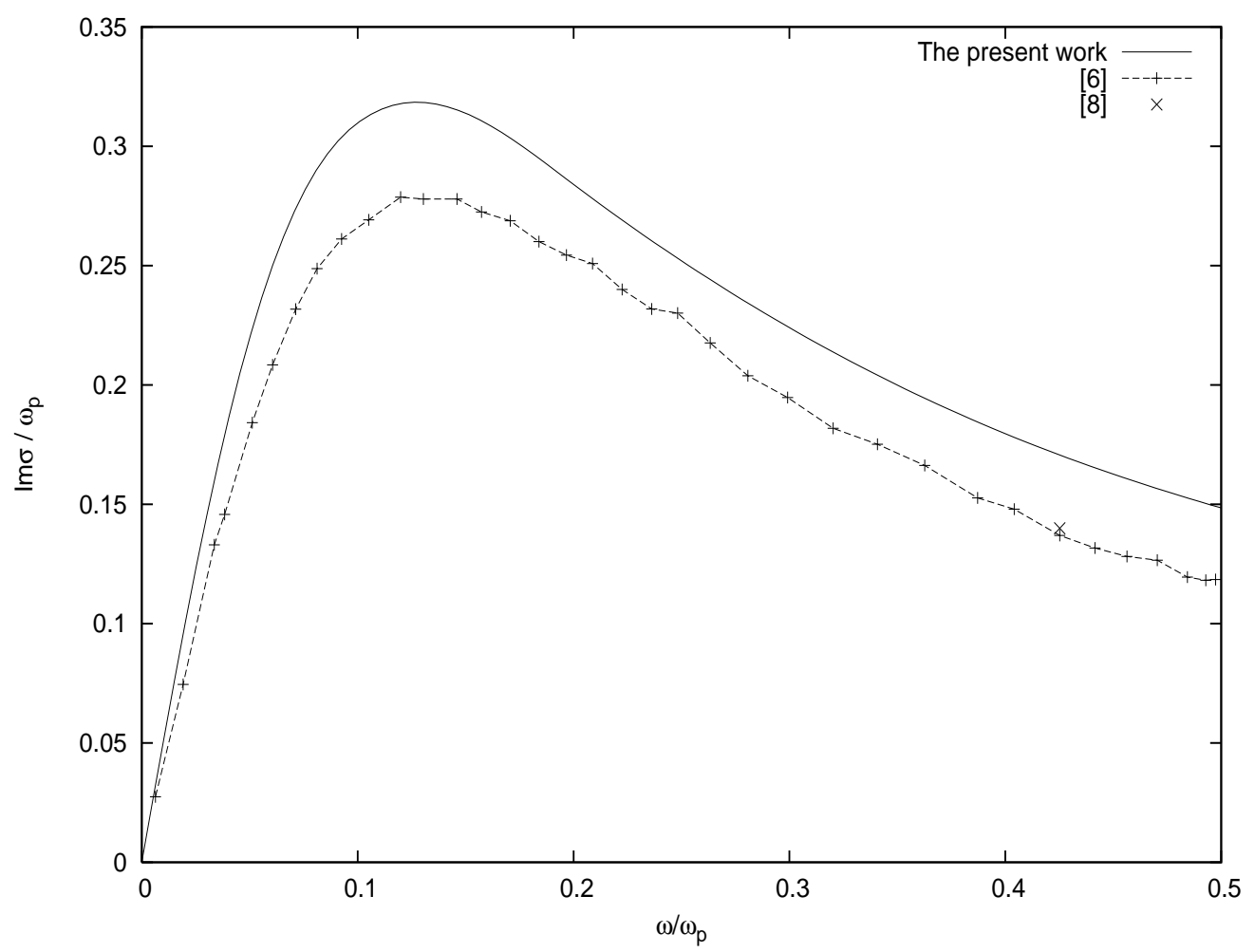

Figure 5: Same as in Fig. 4 but for imaginary part. 


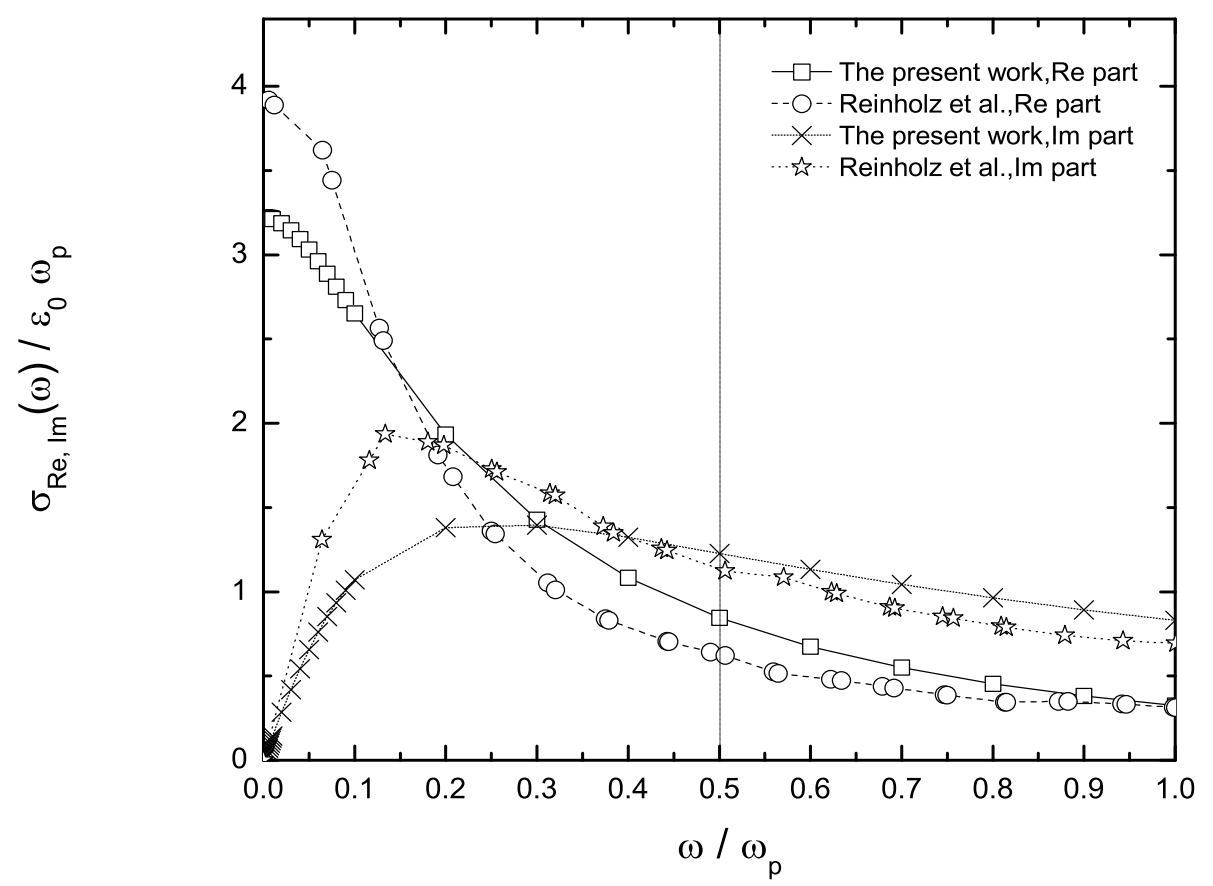

Figure 6: Real and imaginary parts of the plasma dynamic conductivity for $n_{e}=3.8 \cdot 10^{21} \mathrm{~cm}^{-3}, T=3.3 \cdot 10^{4} \mathrm{~K}$, as a function of $\left(\omega / \omega_{p}\right)$ frequency ratio, for $0 \leq \omega / \omega_{p} \leq 1$, together with result from [10]. The value $\omega / \omega_{p}=0.5$ especially mark to remind that within our research the region $0 \leq \omega / \omega_{p} \leq 0.5$ is examined. 


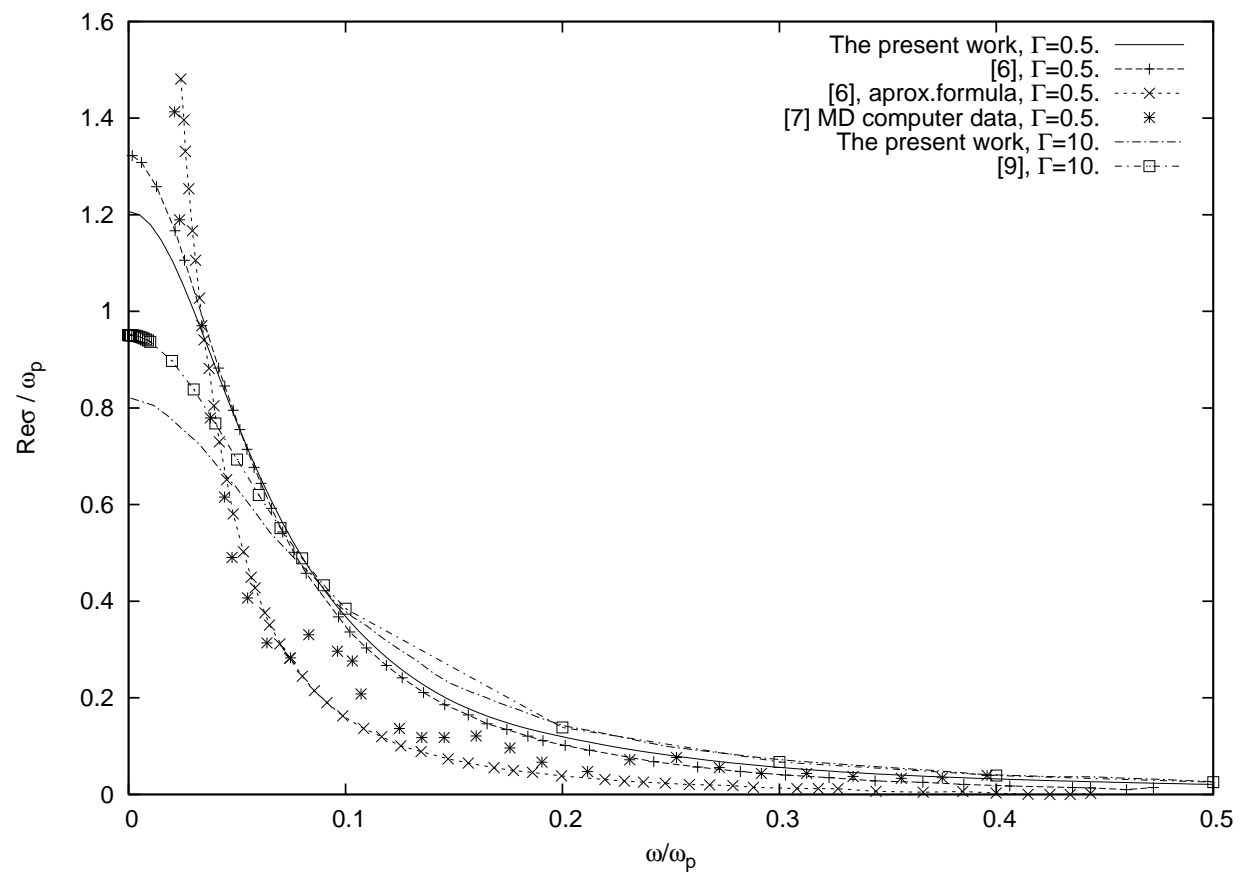

